\title{
Rebuilding Cultural and Heritage Space of Corregidor Island using GPS-Based Augmented Reality
}

\author{
Nancy M. Flores, Lawrence Dolores, Gerald Cayabyab, Thelma Palaoag, Jean Angeles, Gertrude \\ Corpuz, Rhoel Samson, Josephine Dela Cruz, Michelle Mamaril
}

\begin{abstract}
One of the biggest impact of Augmented reality was on the architectural and construction industries. The AR ability to design and construct beyond visualization by virtually walking through the completed model draws inspiration among the researchers the desire to preserve memories behind the war-ravaged buildings that have not been restored, and left as they were after the war in reverence to the Filipino and American soldiers. The software engineering tool, rational unified process, was used as a guide in developing the application with the aid of Android Studio, Unity, Vofuria, Lumions and sketch-up as software development tools.

The application was tested and evaluated by IT experts and tourists of Corregidor using the SUS evaluation tool. Based on the overall satisfaction, usefulness and ease of used among the tourists of Corregidors is "Excellent" which means that the developed mobile applications is more immersive and engaging that will make tourist journey more exciting at the Corregidor Island. This application can assist tourist in experiencing more interactive, fun and engaging journey in Corregidor. Furthermore, this application can served as a mobile tour guide application made for the tourist of Corregidor Island that shall help them learn more about the history and the significance of Corregidor Island by the use of augmented reality to show the original setup of the ruins. The application will help NCCA cascade the historical information about the island and its significance during the war.
\end{abstract}

Index Terms-Augmented Reality, Corregidor Island, GPS-Based Augmented Reality, Cultural and Heritage Space.

\section{INTRODUCTION}

Corregidor Island is one of the most visited tour destinations and said to be a favorite site among foreigners visiting Manila due to the island's fascinating story and

Revised Version Manuscript Received on 10 September, 2019.

Nancy M. Flores, University of the Cordilleras, Philippines. (Email: nm_flores@yahoo.com)

Lawrence Dolores, Technological Institute of the Philippines, Philippines. . (Email: lancedolores@gmail.com)

Gerald Cayabyab, Technological Institute of the Philippines, Philippines. . (Email: gerald.cayabyab@gmail.com)

Thelma Palaoag, University of the Cordilleras, Philippines. (Email: tpalaoag@gmail.com)

Jean Angeles, Technological Institute of the Philippines, Philippines. (Email: jeanmaitem@gmail.com)

Gertrude Corpuz, University of the Cordilleras, Philippines.(Email: gertude_corpuz@yahoo.com)

Rhoel Samson, Technological Institute of the Philippines, Philippines. (Email: archsamson2013@gmail.com)

Josephine Dela Cruz, University of the Cordilleras, Philippines. (Email: delacruzpen@gmail.com)

Michelle Mamaril, University of the Cordilleras, Philippines. (Email: mamarilucchtm@gmail.com) proximity to Metro Manila. It is one of the significant places in the Philippines that played a pivotal role during the Pacific War and liberation of the Philippines from Japanese forces. It was officially called Fort Mills during the American era but was also commonly referred to as "The Rock" for its terrain and fortifications, and "Gibraltar of the East" for its resemblance to Mediterranean Sea's peninsular fortress. Due to its strategic location, it was the biggest of the heavily fortified island that make up the harbor defenses of the capital. Unfortunately, not everyone is aware of the story behind the ruins of buildings, structures, and tunnels in the island of Corregidor(De Mesa, et.al. n.d). Some tourists visiting the place may not recognize its significant role during the Pacific war and liberation of the Philippines. With the help of architectural $3 \mathrm{~d}$ modeling, historical ruins such as buildings, structures, and objects will be seamlessly remodeled and rendered to its original form through augmentation. Likewise, the project hopes to address the lack of public awareness about the island's moving story of a war that claimed so many lives.

Technology has come a long way in presenting new things that may indulge and amuse the masses from communication, lifestyles and cultures, creating a new challenges to historical or heritage sites. From the first creation of the computer to smartphones, and until now we are creating more technological advancements that can help us build a new world that makes life easy. Technology can be applied to make virtual reconstructions or rebuild what was destroyed with time using Augmented Reality or 3D reconstruction.

Augmented reality (AR) is a live, copied view of a physical, real-world environment whose elements are augmented (or supplemented) by computer-generated sensory input Augmented reality takes the real world and adds to it with a 3D model of your design, especially in the field of architecture (Yoders, 2018). Furthermore, Augmented Reality is a technology that the real world with digital information and media such as using Three-Dimensional (3D) models and videos, overlaying in real-time and camera view of your mobile phones, tablets, pc or connected glasses. You can significantly improve its overall functionality with the help of Global Positioning System (GPS) where this satellite-based navigation system made up of at least 24 satellites can be used even in any 


\section{REBUILDING CULTURAL AND HERITAGE SPACE OF CORREGIDOR ISLAND USING GPS-BASED AUGMENTED REALITY}

weather conditions, anywhere in the world, no subscriptions, no fees and to help the application recognize the place where the user stands. The AR can serve as a new platform for future teaching method throughout the world. This can be used to reminisce the fortes of Corregidor Island.

This study covers the development of a mobile application which features innovation, knowledge, portability, and efficiency to the tourist of Corregidor Island. The researchers aim to develop a mobile application that shall help tourist to understand, and learn about the history of Corregidor Island. Using the application, users can have the chance to experience a modern and exciting way of viewing the island, showing a different perspective that will allow new approach on learning the history of Corregidor Island.

\section{REVIEW OF RELATED LITERATURE}

In the study of Change and Chung (2014) those in recent years, hardware and software advances in digital technology have prompted not only the commercial use of augmented reality (AR) systems but also its implementation in education. Marker-based AR systems allow users to input pre-designed markers, and generate new scenes blending the real world and computer-generated 3D images.

In the study written by Okura et al. (2016), they stated that in augmented reality (AR) applications using mobile devices, geometric registration that accurately aligns virtual objects onto real scenes is one of the essential requirements. An augmented image from the device viewpoint is displayed on the mobile device based on real-time camera pose estimation and image-based rendering.

Nevertheless, few AR systems permit simultaneous interactions of multiple markers. This study aims to construct an interactive multimedia learning system. The story script showcases the growth of flowers by applying a series of markers, where interactions with 3D objects can be achieved through AR technology. Learners can use a camera to capture the information of multiple AR markers, and 3D objects and media can be generated on-screen. With variations in AR markers and time changes of the Arduino Clock, one can learn the growth of plants during day and night through a mixed-reality virtual experience that brings fun to learning. Accordingly, this e-learning system is capable of boosting students' learning interests and improving their learning effectiveness.

According to the research of Antonioli et al. (2015) states that today is society, technology has become a crucial part of our lives. It has changed how people think and applied knowledge. One of the newest developing technologies is augmented reality (AR), which can be used to computers, tablets, and smartphones. AR affords the ability to overlay images, text, video, and audio components onto existing pictures or space. If an educator is looking to model scientific practice, AR provides the opportunity to support the multifaceted world of scientific exploration. AR tools are becoming more user-friendly and require less programming skills making them more attractive to the ordinary educator. Teachers are concerned with the programming and coding that is necessary to integrate AR activities into their classrooms. The software is being developed (i.e., The Art of Illusion) for teachers to focus on building educational content and not having to worry about programming skills
(Billinghurst and Dunser, 2012).

In a study written by Wei et al. (2016), AR technology can involve superimposing virtual scenarios over real environments and achieving fantastic yet authentic effects, its application in building authentic learning environments to realize authentic activities has attracted an increasing number of researchers. It can provide a reasonable level of realism and interactivity as well as life-like situated learning experiences; Game-based learning is thought to be an effective tool for learning that can promote the enhanced learning experiences and student motivation.

According to Durairaj and Aurelia (2017), the real world with virtual data embedded in it is allowed in augmented reality. The user's understandability and perception are increased by mixed reality. Location-based services, GPS compass, portability, versatility and enhanced technology based on usability are the remarkable advantages of mobile augmented reality. A mobile augmented reality based active learning system provides a modified level of usability and learning because it is suitable for any environment, experience or situation.

As stated by Webster et al. (2017) developing of augmented reality systems to prove methods for the construction, inspection, and renovation of architectural structures. Augmented reality systems add virtual computer-generated material to the surrounding physical world. Our augmented reality systems use see-through head-worn displays to overlay graphics and sounds on a person is naturally occurring vision and hearing. As the person moves about, the position and orientation of his or her head are tracked, allowing the overlaid material to remain tied to the physical world. We describe an experimental augmented reality system that shows the location of columns behind a finished wall, the area of re-bars inside one of the columns, and structural analysis of the column. We also discuss our preliminary work in developing an augmented reality system for improving the construction of space frames.

The similarities of the researches of Change and Chung (2014), Antonioli et al. (2015), and Webster et al. (2017), and our research study, an application that will apply the revolutionary way of integrating Augmented Reality to your surroundings, only by using smartphones it will allow overlay graphics to materialize in the physical world with the use of camera. The difference between our studies is that the tourists of Corregidor Island will use our application as they need a new and exciting way to explore and learn about the island, bringing a new perspective and at the same time allowing a new potential use for Augmented Reality.

Several Studies and research areas of Norsyafawati et al. (2015) stated that the significant milestones in mobile Augmented Reality between 1968 and 2014. Mobile Augmented Reality has primarily evolved over the last decade, as well as the interpretation itself of what is Mobile Augmented Reality. The advances of mobile technology, anyone who uses mobile devices such as a smartphone, iPad, and webcam can experience it. By using AR, an application is 
designed inside your tablet which is similar to the live view of the physical world. The objective of this study is to review related literature that could highlight several significant knowledge gaps for a new exploration of Augmented Reality study. With the transformation and miniaturization of physical devices and displays, the concept of mobile Augmented Reality evolved towards the notion of "mobile device," aka Augmented Reality on a mobile device. In this history of mobile Augmented Reality, we considered both definitions and the evolution of the term over time.

According to Acharya A. (2014), some of the main depth related perceptual issues in augmented reality on a range of different platforms. While the problems ranged from conflict of cues due to incorrect rendering to ergonomics, each platform is tailored to some specific issues. This draws our attention to the fact that solutions to the problem have to be specific to the platform than a general one. We focused our attention more on the perceptual impact of motion in an environment where a change in position can influence the dominating cue. Many empirical pieces of evidence have been shown with regards to shadows being a useful cue to perceive the spatial relationship of an object with its environment correctly. But, looking from a psychological perspective, we found more unanswered questions than solutions. There remains a massive gap in understanding this phenomenon from a psychological perspective.

Studies conducted by Chatterjee and Chandran, (2017); Ahmed et al., (2014), stated that in the Kanade-Lucas-Tomasi tracker is face detection and tracking is one of the emerging research areas in the image analysis and computer vision systems. This face detection and tracking help local security forces to investigate crime incidents. Kanade-Lucas-Tomasi tracks the features from one image to the next. The features are scattered throughout the image, depending upon the overall texture of the background and the number of objects in the picture.

The main challenge comes in KLT tracker implementation is how to select the appropriate window size that is suitable for accurate tracking.

The researches of Norsyafawati et al. (2015), Acharya A. (2014), Chatterjee and Chandran, (2017); Ahmed et al., (2014), states that perceptual impact on motion will be necessary because the connection between the camera and its movement will be essential to execute a successful augmented reality project. It also states that we need to consider psychological perspective to understand the useful cue for correct perceive spatial relation of an object to its environment, making sure that the augmented object will be appropriate for the right surroundings. These statements have similarity in our study, which is to design an application that will provide a new interactive way of exploring Corregidor Island for the tourist. The difference between the studies and ours is that it focused on the camera's perception and not on the user's perspective.

\section{METHODOLOGY}

\section{A. Overall Strategy}

The developed application enhances the tourists experienced using a Mobile Augmented Reality for Corregidor Island application that immerses users in the virtual reconstruction of a cultural site by showing how the location looked at different periods in history. The 2D/3D models of old buildings are rendered in their original locations and overlaid on the real world view, acquired with the device camera. Therefore, user can wander around to see the buildings from every point of view and navigate in a space where real and virtual elements coexist.

Historical multimedia information related to the places visited can be accessed from the application by simply tapping on the displayed objects. This includes augmented objects such as buildings, objects, video clips, and other related events that can be augmented. It helps tourists in accessing valuable information and improving their knowledge regarding a touristic attraction while enhancing the tourist experience and offering increased levels of entertainment throughout the process. Such information systems are able to personalize the delivery of the multimedia content according to the user's characteristics and the use context, thus supporting their deployment for a number of scenarios. This freedom of interaction with the application and with the available historical contents, allows tourists to play an active role in their journey through the application. The development of the systemswas guided using the Rational Unified Process as shown below:

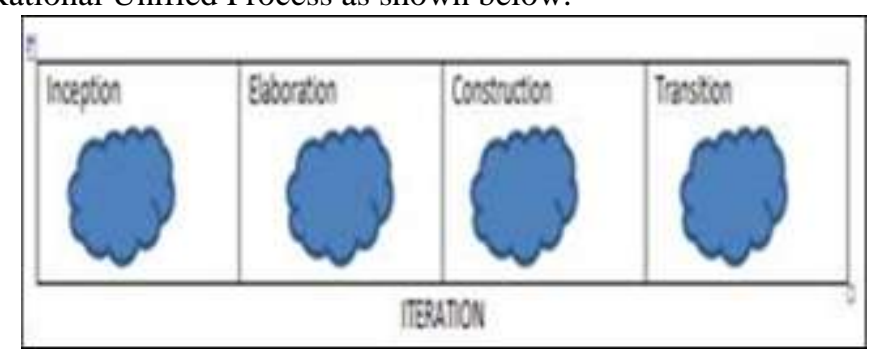

Figure 1: Rational Unified Process

The system is designed to handle multiple $2 \mathrm{D}$ and $3 \mathrm{D}$ content, in order to provide tourists a true timeline of the evolution of Corregidor. Figure 2 shows how the system works in vofuria.

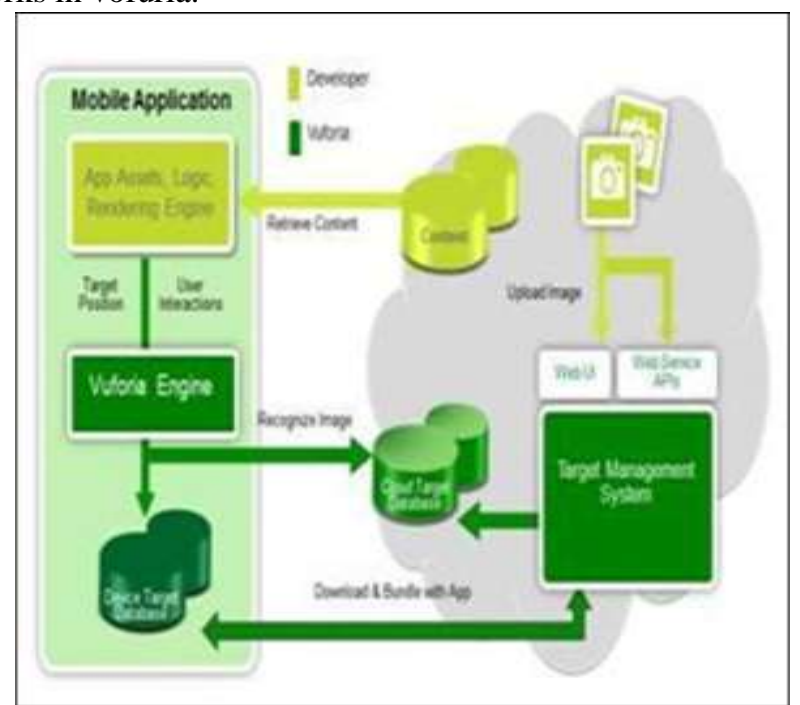

Figure 2: Application development process with the Vuforia Platform 


\section{REBUILDING CULTURAL AND HERITAGE SPACE OF CORREGIDOR ISLAND USING GPS-BASED AUGMENTED REALITY}

The application development process with the Vuforia Platform and is based on the Vuforia Cloud Recognition System which enables our application to see or recognize images using cloud database. Vuforia is a software platform that uses top-notch, consistent, and technically resourceful computer vision-based image recognition and offers a wide set of features and capabilities, giving developers the freedom to extend their visions without technical limitations.

The application utilizes the mobile phone's camera to scan the target image which will be then rendered using Unity Engine. Through the internet, the application will connect to the Vuforia Cloud Server to find a match in the database. If a match is found, the server will send a response and command the engine to render an active $3 \mathrm{~d}$ text which will serve as an interaction cue for the users. Upon touching/clicking the $3 \mathrm{~d}$ text, the application will now display all the related information on the image. On the instance that there is no network connection available, the application has a search engine that can be used to manually search for names of historical sites.

\section{RESULTS AND DISCUSSIONS}

\section{A. Systems Architecture}

The systems architecture presented in figure 3 shows how tier architecture was used to illustrate how the system works. The first tier is the presentation layer where the user starts exploring the features and capability of the application. Second, the logic tier where the application used the Kanade-Lucas-Tomasi Algorithm that shall significantly clearer way of using it while enhancing image quality, it shall also be able to recognize object or image target to let Vuforia know that the target will be used for augmenting, and locate or track the Longitude and the Latitude for the GPS to work. Lastly, the Data tier is where the application will fetch the images and models that are going to be used for augmented reality.

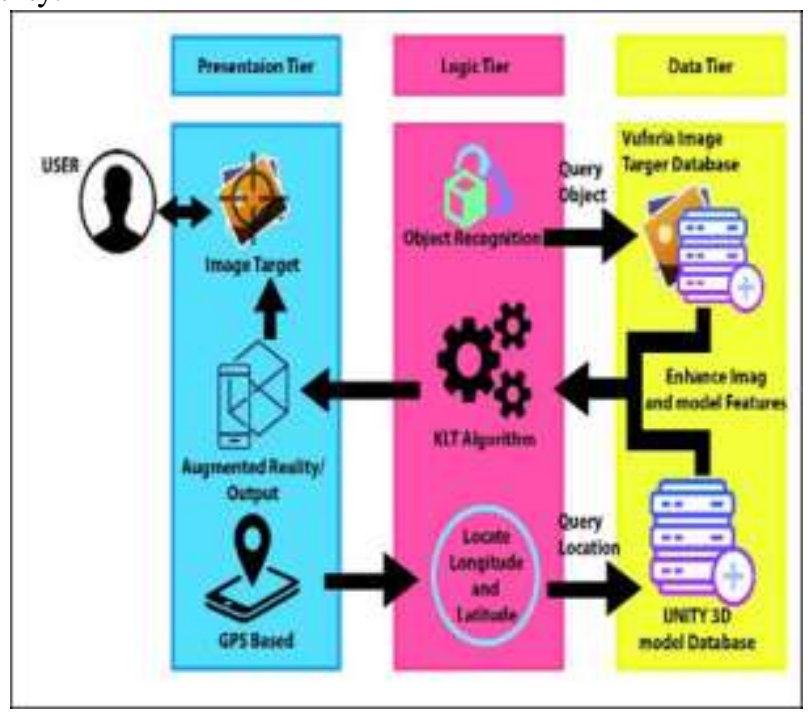

Figure 3: Systems Architecture

\section{B. Systems Features}

CorregiTour is a user-friendly application that can be used easily with the target users. The color yellow-orange and brown is used to make the application looks vintage since the the application is presented and how it functions. The three improve the motion of the camera, allowing for a smooth and

application is about History. In the home as shown in Figure 4 , it is where you can find the features of the application such as augmented reality camera, maps, explore Corregidor, images, and videos.

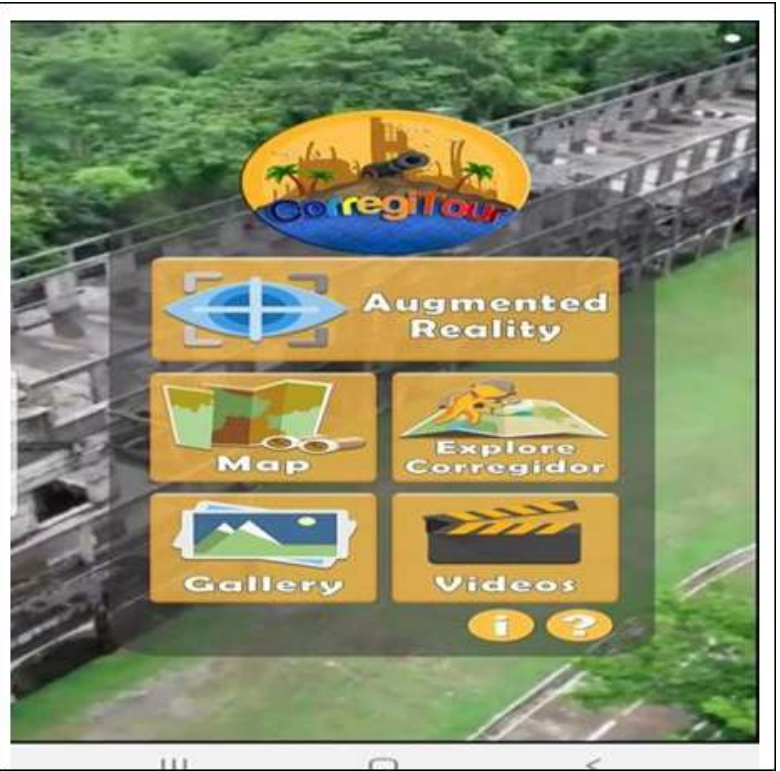

Figure 4: Home Screen of CorregiTour

\section{B.1 Augmented Reality}

Figure 5 shows the augmented reality, where it contains the models and animations that will show the original setup of the ruins in the Corregidor Island. The developer used sketch up and blender for creating the models and animations, then import it to unity and vuforia where they set target images for every models and export it to be able to implement it the Android studio to make it visible to the mobile application.

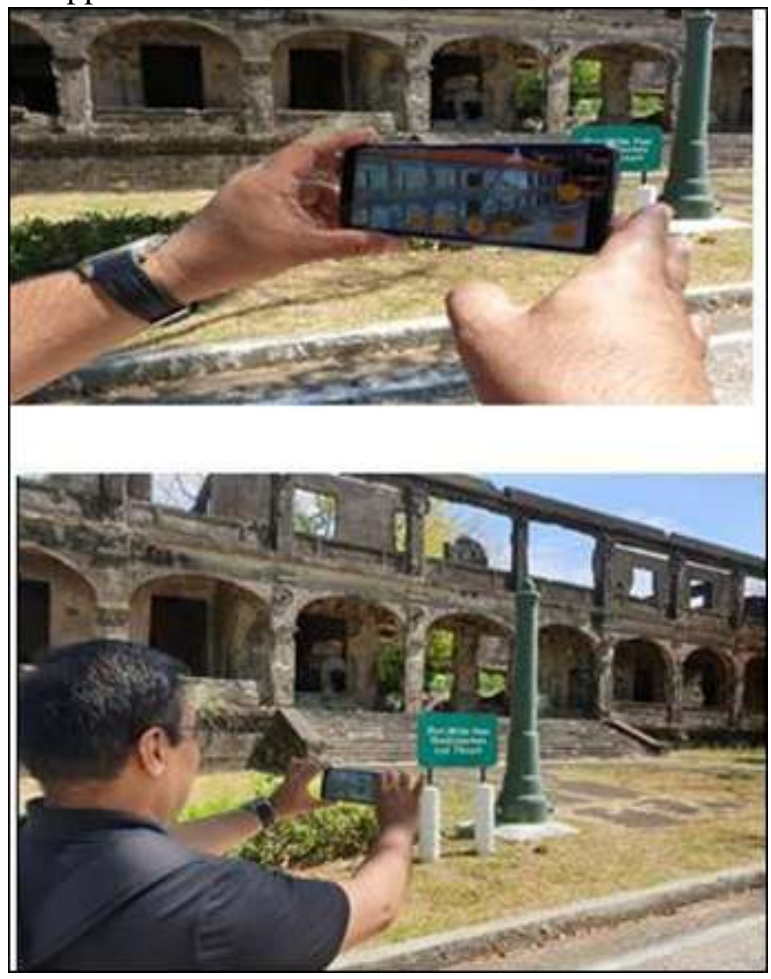

Published By: 


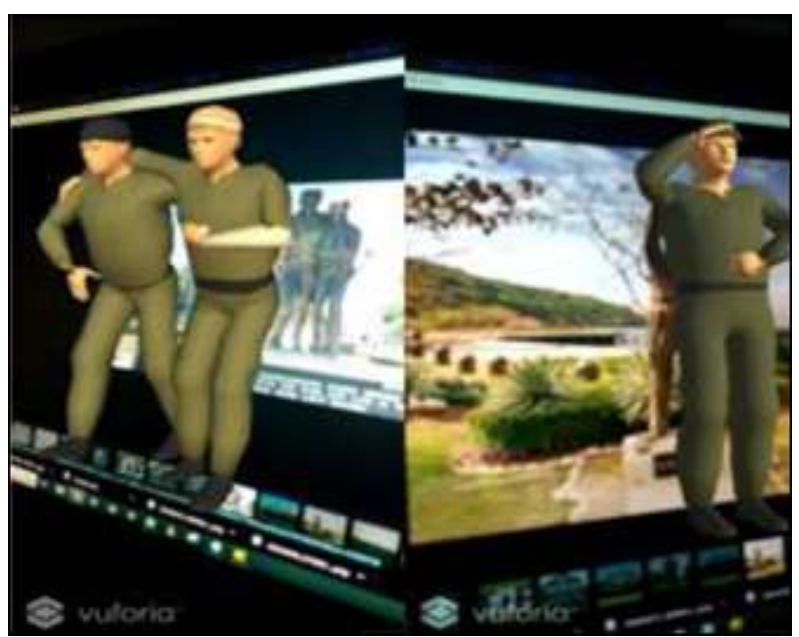

Figure 5: Augmented Reality

\section{B.2 Maps}

Figure 8 shows the Maps module. Image 1 shows the users and for the image 2 , it shows the street view of the structures in the island. The developer used Google Maps for the maps module and get the longitude and latitude of every structure, and set a pin for its specific information. The developer used the card view to display the buttons for the functionalities.

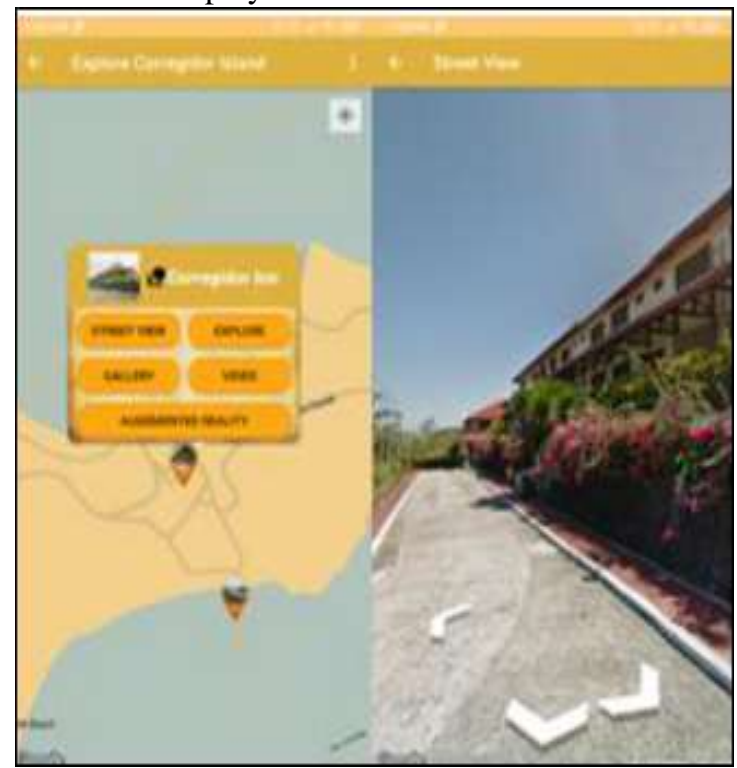

Figure 6: Maps

\section{B.3 Explore Corregidor}

Figure 7 illustrates the Information about Corregidor Island. In the first image, it shows the list of topics that the user can browse. The developer uses linear layout and scroll view to create the view of the list of topics. The second image shows the information about Corregidor, it has text, images, and audio where audio can translate language that is English and Japanese. The developer used card view, text view for the text, image view for the images and a fab button for the audio or text to speech. The contents of the information that were present on the application were validated by Dr. Augusto V. De Viana, a historian and academician of UST and authors of Philippine history books .Likewise, the Japanese translation of the text in the application were transcribed by Ms. Rowena P. Okabe, President of the Association of Nihungo Teachers-Northern Luzon. position and the position of the point of interest in Corregidor
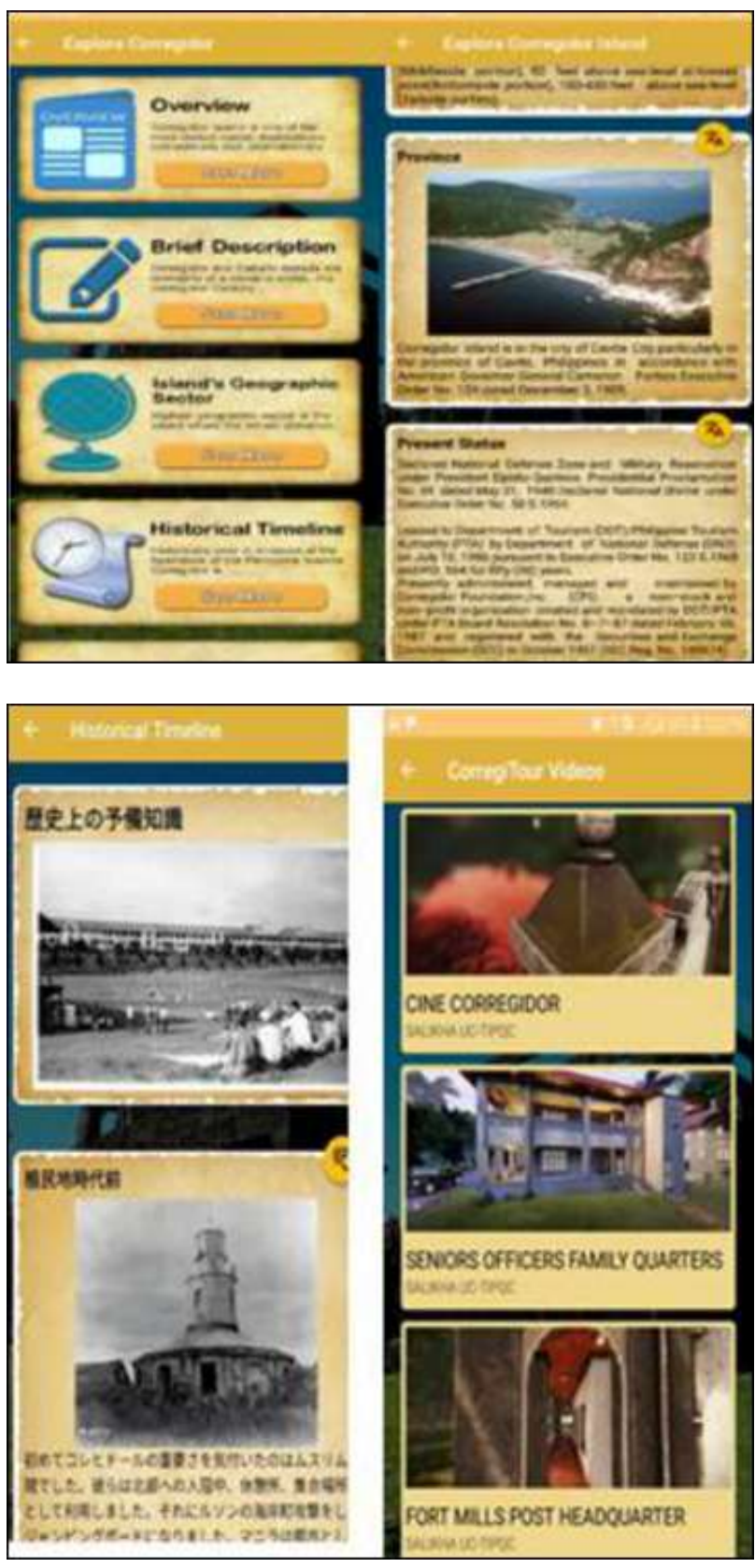

Figure 6: Maps

\section{V.CONCLUSIONS}

Rebuilding the cultural and heritage space of Corregidor Island using GPS-Based Augmented Reality enriches the teaching and learning experience of the target users especially in the area of historical and cultural heritage specifically in Corregidor Island. The application "CorregiTour "was developed to help the National Commission for Cultural and the Arts to promote the rich history of Corregidor Island, providing a virtual tour guide in Corregidor Island that they can appreciate the great infrastructure before the World War II.

Likewise, the developed application shall also increases the potential awareness and effectiveness in knowledge acquisition by creating a medium that uses a creative and technology based user experience learning. This undertaking

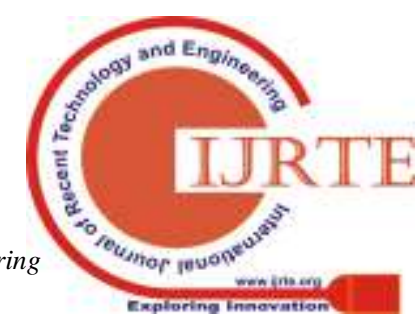




\section{REBUILDING CULTURAL AND HERITAGE SPACE OF CORREGIDOR ISLAND USING GPS-BASED AUGMENTED REALITY}

is set forth to be utilized as a dynamic development to human interaction with its immediate environment as part of a promotional pursuit of the historical events and cultural heritage of Corregidor Island being the pilot project. This shall also consign the Philippine Tourism industry in a better stature. This project plays an important role in the advancement of national tourism, heritage and arts by means of Augmented Reality (AR) that shall contribute to national identity. It certainly enrich higher education, provide economic development, profit and jobs to the community. This shall be a fundamental factor for image building for any tourist destination in the Philippines.

\section{ACKNOWLEDGEMENT}

The researchers' sincerest gratitude to all the following people who have academically and technically contributed to the realization of this document.

The administration of the University of the Cordilleras and Technological Institute of the Philippines-Quezon City for their endorsement and unconditional support.

The officials and staff of the Commission on Higher Education and National Commission for Culture and the Arts for their approval and monitoring of this project.

The employees in related government agencies and offices, for their insights and linkage especially to Yssa Martinez, Tourism Infrastructure and Enterprise Zone Authority, Rowena Bautista, Corregidor Foundation, Mona Lisa Lauron, National Historical Commission of the Philippines and Riah G. Almodovar, Travel People.

Dr. Agusto V. Deviana, historian and academician, University of Santo Tomas for the validation of the historical research content and Rowena P. Okabe, president association of Nihongo Teachers-Northern Luzon for the English to Japanese historical research content translation used for the language translation feature of the project.

The researchers greatly benefited from the panel of experts, Dr. Ma. Mercedes T. Rodrigo and Dr. Marinett Vega for the reviews beyond reproach which made the project a project of careful scrutiny in coming up with a scholarly document.

We solemnly give praises to the Almighty for the graces and blessings of supportive family and friends from whom we derive all our inspiration, strength and enlightenment.

\section{REFERENCES}

1. Change and Chung (2014). The interaction of child-parent shared reading with an augmented reality (AR) picture book and parents' conceptions of AR learning

2. Anthony W., Steven F., William M. (2016) Augmented Reality in Architectural Construction, Inspection, and Renovation

3. Misty A., Corinne B., and Kelly S. (2015). Augmented Reality Applications in Education pp. 96-97.

4. Fatin N., et al. (2016). An Exploratory Study on Mobile Augmented Reality (AR) Application for Heritage Content. Faculty of Technology Creative \& Heritage, University Malaysia Kelantan, Bachok, Malaysia.

5. Fumio O., Yuya N., Tomokazu S. (2016). Motion Parallax Representation for Indirect Augmented Reality

6. Kris K. Kanade-Lucas-Tomasi (KLT) Tracker Debmalya C., Saravanan C. (2016). Comparative study of the camshaft and KLT algorithms for real-time face detection and tracking applications
7. Manoj D., P. Sagaya A. (2017). A modernistic and contemporary mobile augmented reality erudition system. 2017 International Conference on Computing Methodologies and Communication (ICCMC)

8. Wei F., Lianyu Z., Huanjun D., and Hongbo Z. (2017). Real-Time Motion Tracking for Mobile Augmented/Virtual Reality Using Adaptive Visual-Inertial Fusion.

9. Dr. Tasneem A. (2014). Application of KLT (Kanade-Lucas-Tomasi) Tracker for Hotspot Observation. Conference: geomatrix'14 National Conference on Geo-informatics in Rural, Urban and Climatic Studies, At IIT Bombay.

10. Jeff Yoders, (2018). What is Augmented Reality and how can It help Architects and Contractors?

11. De Mesa, D. M., Rosel, D., Montoya, S., Camama F.,Sugatan, D., Gumapas, L. (n.d) The history and cultural life of Cavite Province. Philippines: Historical Data Bank. National Historical Institute Library. (pp 11-12)

12. Corregidor Foundation Inc. - Brochure WALK-IN Loop Tour (n.d.)

13. Medina, I. R. 2002. Cavite before the revolution (1571-1986). Quezon City:Cavite Historical Society \& the University of the Philippines Press.(p. 153)

14. Combat History Division G-1 Section. (n.d.). Corregidor of eternal memory. (pp.19-20)

15. Camagay, M.L. T, Ancheta J.A.,Bernal M.,Guiang, F.J.P.A., Malban, F.J.M.,Ramos, D.P.G. 2018 Unraveling the past: readings in Philippine history. Quezon City: Vibal Group Inc. (p193) 